\title{
Doppler Tisular: velocidad de desplazamiento miocárdico en sístole en pacientes con hipertensión arterial con fracción de eyección conservada
}

Katia Galdames ${ }^{1}$, Jorge Torres ${ }^{1}$, María Ester Goldsack ${ }^{1}$, Francisco Piñats ${ }^{1}$, Felipe Solar ${ }^{2}$, Felipe Olivaresa, Paz Tabiloa, Andrés Pobletea.

1 Laboratorio de Ecocardiografía, Hospital Militar de Santiago, Santiago de Chile.

2 Departamento de Epidemiología y Salud Pública, Universidad de los Andes, Santiago de Chile.

a Alumnos de medicina, Universidad de Los Andes.

Antecedentes: El doppler tisular podría mejorar la evaluación pronóstica en pacientes hipertensos con función sistólica normal o aparentemente normal como en la disfunción diastólica. Valores bajos de velocidad de desplazamiento miocárdico en sístole ( $\mathrm{Sm}$ ) están asociados con deterioro de la función sistólica, cardiopatía hipertensiva y mortalidad de causa cardíaca. No obstante, la asociación de la onda Sm con parámetros de aumento de presión de fin de diástole aún no es clara.

Objetivos: Estimar la asociación entre la onda Sm y diferentes parámetros ecocardiográficos morfológicos, de función sistólica y diastólica en pacientes con hipertensión arterial que poseen fracción de eyección conservada.

Métodos: En un análisis retrospectivo de 1198 pacientes hipertensos (con fracción de eyección preser- vada) entre los años 2007 y 2010, se evaluó onda Sm y su asociación con la fracción de eyección, índice de masa ventricular (IMV), volumen de aurícula izquierda indexado (VAI), presión de arteria pulmonar en sístole (PAPS) y la relación E/e'.

Resultados: Se observó una tendencia al descenso de la onda Sm a medida que aumenta el IMV $(\mathrm{p}<0,01)$. De los pacientes con $\mathrm{Sm}<6 \mathrm{~cm} / \mathrm{s}$, el 76,6\% de presentó E/e' $\geq 13 \mathrm{~cm} / \mathrm{s}(\mathrm{p}<0,01)$. Además, Sm normal se asoció con $\mathrm{E} / \mathrm{e}^{\prime}<13 \mathrm{~cm} / \mathrm{s}$, VAI $<32 \mathrm{ml} / \mathrm{m} 2$ y PAPS $<35 \mathrm{mmHg}$ en un $93 \%, 71 \%$ y $92 \%$ respectivamente $(\mathrm{p}<0,01)$.

Conclusiones: La medición de la onda Sm aporta valiosa información, no sólo de función sistólica, sino también de función diastólica, en pacientes hipertensos con hipertrofia ventricular izquierda.

\section{Correspondencia:}

Nombre: Katia Alejandra Galdames Ibáñez

Dirección: Avenida Larraín 9100, La Reina

Correo electrónico: kgaldames@vtr.net 


\section{Tissue Doppler - myocardial systolic velocity in patients with arterial hypertension and preserved ejection fraction}

Background: Tissue Doppler imaging (TDI) could improve the prognostic evaluation of patients with arterial hypertension in whom systolic function is normal as well as in those with diastolic dysfunction. Low values of peak systolic myocardial velocity $(\mathrm{Sm})$ are associated with systolic function impairment, hypertensive cardiomyopathy and mortality from cardiac causes. Nevertheless, the association of Sm wave with increased enddiastolic pressure is not clear yet.

Aim: to estimate the correlation between Sm wave and several echocardiography morphologic and functional parameters in patients with arterial hypertension and preserved ejection fraction.

Methods: in a retrospective analysis (2007-2010) of 1198 hypertensive patients with preserved ejection fraction, Sm wave was evaluated and corre- lated with ejection fraction, left ventricular mass index (VMI), left atrium indexed volume (LAIV), pulmonary artery systolic pressure (PAPs) and E/e' ratio.

Results: a decrease Sm was observed as VMI increased $(\mathrm{p}<0.01)$. Among patients with $\mathrm{Sm}<6 \mathrm{~cm} / \mathrm{s}$, $76.6 \%$ had E/e' $>13(\mathrm{p}<0.01)$. Normal $\mathrm{Sm}$ values were observed in $93 \%$ of patients with $\mathrm{E} / \mathrm{e}$ ' ratio $<13,92 \%$ of patients with LAID $<32 \mathrm{ml} / \mathrm{m} 2$ and $71 \%$ of patients with PAPs $<35 \mathrm{mmHg}(\mathrm{p}<0.01)$.

Conclusion: Sm wave measurement gives valuable information about systolic function as well as enddiastolic pressure in hypertensive patients with left ventricular hypertrophy.

Keywords: Doppler echocardiography, left ventricular hypertrophy, ejection fraction.

\section{Introducción}

La hipertensión arterial (HTA) constituye un problema a nivel mundial y nacional, siendo el principal factor de riesgo para enfermedad coronaria y cerebrovascular ${ }^{1}$. Aunque su prevalencia ha disminuido, se estima que uno de cada tres personas la padece, el 34,9\% desconoce su condición y solamente el $16,5 \%$ tiene un control óptimo de presión arterial $^{2}$.

El Doppler Tisular (DT) podría complementar a la ecocardiografía convencional en la evaluación del paciente hipertenso. Wang et al. demostraron que parámetros del DT superaban a los de ecocardiografía convencional al momento de predecir resultados adversos en pacientes cardiópatas. Valores de $S m \leq 5 \mathrm{~cm} / \mathrm{s}$ se asociaron mayor mortalidad cardíaca, la que fue aún mayor con $S m \leq 3 \mathrm{~cm} / \mathrm{s}^{3}$. Posteriormente, Shin et al. observaron retrospectivamente mayor tasa de eventos cardiovasculares adversos en pacientes con $\mathrm{Sm}<5 \mathrm{~cm} / \mathrm{s}$ en 148 pacientes con fibrilación auricular y FE conservada ${ }^{4}$. Estudios adicionales apoyan el valor pronóstico de parámetros del DT en pacientes con insuficiencia cardíaca, IAM e HTA ${ }^{5-7}$. Pese a lo anterior, existe limitada evidencia que sustente específicamente su uso para evaluar función diastólica, siendo la relación E/e' el principal parámetro asociado a la presión de fin de diástole (PFD) 8. Valores de E/e' $\leq 8$ predicen PFD conservada, mientras que E/e' $\geq 13$ se asocia a aumento en la PFD. Sin embargo, cuando se obtiene un valor intermedio, es decir entre 9-13, esta asociación disminuye y se requieren otros parámetros complementarios, entre ellos el VAI y PAPS $^{8}$. De acuerdo con las Guías de Sociedad Americana de Cardiología valores intermedios de E/e' predicen PFD normal cuando el VAI es menor a $34 \mathrm{ml} / \mathrm{m} 2$ y la PAPS es menor a $30 \mathrm{mmHg}^{9}$. Valores normales de Sm podrían asociarse a PFD conservada, por lo que determinar su asociación podría ser una herramienta adicional en este subgrupo de pacientes. Finalmente, no existe evidencia en 
nuestro medio que evalúe su utilidad como marcador precoz de hipertrofia ventricular izquierda (HVI). El objetivo de este trabajo es determinar asociación entre la onda $\mathrm{Sm}$ y diferentes parámetros ecocardiográficos morfológicos, de función sistólica y diastólica en pacientes hipertensos con fracción de eyección preservada.

\section{Método}

Se realizó un análisis retrospectivo a 1198 pacientes hipertensos derivados para Ecocardiograma entre los años 2007 y 2010, en Hospital Militar de Santiago de Chile. Se incluyeron aquellos con FE mayor a $50 \%$.

El examen fue realizado por 4 operadores, con experiencia tanto en la técnica convencional, como en el doppler tisular.

Se utilizó un Equipo GE Vivid 7, transductor 3.5mhz y Philips Ie33.

Mediante la técnica en Modo M se realizó la medición de diámetros y grosor de pared septal y posterior en Eje largo paraesternal. Se estimó el IMV por Fórmula de Devereaux. Mediante técnica 2D se estimó el volumen de aurícula izquierda indexado en 4C y $2 \mathrm{C}$ apical y se estimó PAPS de acuerdo al máximo gradiente transtricuspideo y presión auricular derecha.

Mediante doppler tisular se realizó la medición de la onda $\mathrm{Sm}$, promediando el anillo mitral septal y lateral. Se determinó su asociación con la FE, la cual fue obtenida mediante el método de Simpson. Además, se determinó su asociación con el VAI, el IMV, la PAPS y la relación E/e', (promediando el anillo mitral septal y lateral).

Para medir la relación entre dos variables continuas, se utilizó el coeficiente de correlación de Pearson (r), y para las variables cualitativas la prueba de chi-cuadrado. La diferencia de medias se analizó con la prueba t-student. Se consideró una significancia estadística del 5\% ( $\mathrm{p}<0,05)$.

Las mediciones fueron obtenidas de la base de datos del Laboratorio de Ecocardiografía, del Hospital Militar de Santiago y los datos fueron analizados mediante el programa computacional STATA 11.0.

\section{Resultados}

De 2063 hipertensos referidos para ecocardiografía, 1198 tenían FE mayor a 50\% y determinación de la onda Sm y fueron incluidos en el análisis. La edad promedio fue de 66 años (DS 13.4), el 54\% eran mujeres y la FE promedio fue de $66 \%$ (DS 6,0) (Tabla 1).

Un $29,3 \%$ presentó $\mathrm{Sm}<7,5 \mathrm{~cm} / \mathrm{s}$ y no existió correlación estadísticamente significativa entre los valores de FE y onda $\operatorname{Sm}(r=0,09, \mathrm{p}<0,01)$.

Se observó una tendencia significativa al descenso de la
Tabla 1: Características de la muestra

\begin{tabular}{|c|c|c|c|c|c|}
\hline Variables & Promedio & DS & Mediana & Min & Máx \\
\hline Edad (años) & 66,18 & 13,5 & 67 & 18 & 96 \\
\hline FE (\%) $\quad 66,55$ & 6 & 66,5 & 51 & 85 & \\
\hline $\mathrm{Sm}$ (cm/seg) & 8,45 & 1,9 & 8 & 3 & 15 \\
\hline E/e' $\quad 9,68$ & 4,8 & 8.6 & 3.4 & 53 & \\
\hline $\operatorname{VAl}(\mathrm{ml} / \mathrm{m} 2)$ & 30,96 & 9,3 & 29 & 13 & 74 \\
\hline PAPS (mmHg) & 29,34 & 6,5 & 28 & 16 & 70 \\
\hline IMV (g/m2) & 108,91 & 31,2 & 105 & 50 & 292 \\
\hline
\end{tabular}

FE: Fracción de eyección

Sm: Velocidad de desplazamiento miocárdico en sístole

VAI: Volumen atrio izquierdo

PAPS: Presión media de arteria pulmonar en sístole

IMV: Índice de masa ventricular

DS: Desviación estándar

onda $\mathrm{Sm}$ a medida que aumenta el IMV, con valores de $\mathrm{Sm} \geq 7,5 \mathrm{~cm} / \mathrm{s}(\mathrm{p}<0,01)$. Cuando se analizó esta asociación en el subgrupo con Sm alterado, se observó que la tendencia fue estadísticamente significativa en hombres $(\mathrm{p}<0,01)$ (Tablas 2 y 3 ).

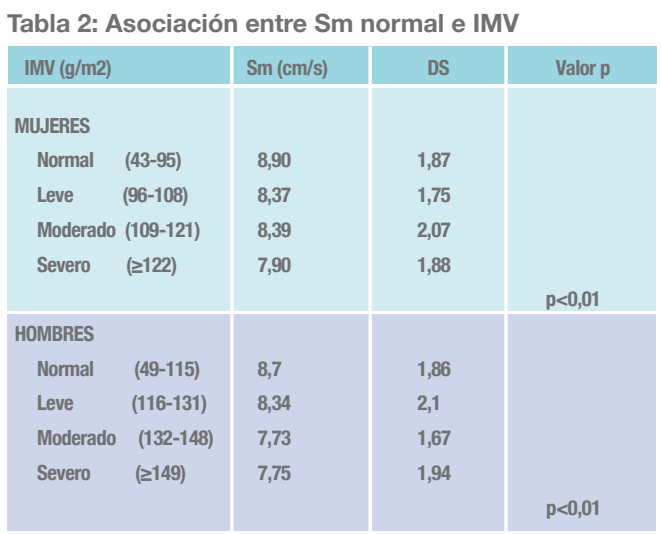

IMV: Índice de masa ventricular

Sm: Velocidad de desplazamiento miocárdico en sístole

DS: Desviación estándar

Tabla 3: Asociación entre Sm alterado y aumento del IMV

\begin{tabular}{|c|c|c|c|}
\hline IMV (g/m2) & Sm (cm/seg) & DS & Valor $p$ \\
\hline \multicolumn{4}{|l|}{ MUJERES } \\
\hline Normal (43-95) & 6,43 & 0,78 & \\
\hline Leve $\quad(96-108$ & 6,37 & 0,84 & \\
\hline Mod $\quad(109-121)$ & 6,42 & 0,78 & \\
\hline Severo ( $\geq 122)$ & 6,19 & 0,92 & \\
\hline & & & $p=0,057$ \\
\hline \multicolumn{4}{|l|}{ HOMBRES } \\
\hline Normal (49-115) & 6,44 & 0,78 & \\
\hline Leve (116-131) & 6,27 & 0,86 & \\
\hline Mod $\quad(132-148)$ & 6,18 & 1,03 & \\
\hline Severo ( $\geq 149$ ) & 6,15 & 0,87 & \\
\hline & & & $p<0,05$ \\
\hline
\end{tabular}

IMV: Índice de masa ventricular

Sm: Velocidad de desplazamiento miocárdico en sístole

DS: Desviación estándar 
En los pacientes con relación E/e' $\geq 13$, el 71,7\% tuvo Sm alterada y se comprobó asociación ( $\mathrm{p}<0,01)$. De los pacientes con $\mathrm{Sm}<7,5 \mathrm{~cm} / \mathrm{s}$, sólo el 40,5\% presentó E/e' mayor o igual a 13. Sin embargo, este porcentaje aumenta al disminuir el nivel de corte de la onda $\mathrm{Sm}$ a $<6 \mathrm{~cm} / \mathrm{s}$, con el 76,6\% de los pacientes presentando E/e' mayor o igual a $13 \mathrm{~cm} / \mathrm{s}$, siendo esta asociación significativa $(\mathrm{p}<0,01)$

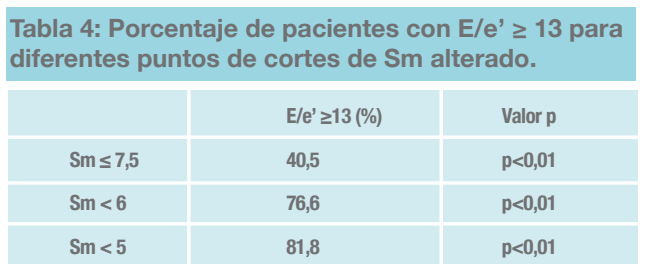

Sm: Velocidad de desplazamiento miocárdico en sístole.

\section{(Tabla 4).}

Valores normales de onda Sm se asociaron con E/e' menor a 8 y 13 , en $57.6 \%$ y $93.9 \%$ respectivamente $(\mathrm{p}<0,01)$. Además, se observó asociación de Sm normal con VAI $<32 \mathrm{ml} / \mathrm{m} 2$ y PAPS $\leq 35 \mathrm{mmHg}$ en un $71 \%$ y $92 \%$, respectivamente, todos con valores de $\mathrm{p}<0,01$. Finalmente, valores de $\mathrm{Sm} \geq 7.5 \mathrm{~cm} / \mathrm{s}$ se asociaron en un $64,8 \%$ a relación $\mathrm{E} / \mathrm{e}^{\prime}<13, \mathrm{VAI}<32 \mathrm{ml} / \mathrm{m} 2$ y PAPS $\leq 35 \mathrm{mmHg}(\mathrm{p}<0,01)$ (Tabla 5).

\begin{tabular}{|c|c|c|}
\hline \multicolumn{3}{|c|}{$\begin{array}{l}\text { Tabla 5: Asociación entre Sm normal y parámetros } \\
\text { de aumento de presión diastólica final. }\end{array}$} \\
\hline & $\mathrm{Sm} \geq 7.5$ & Valor $\mathbf{p}$ \\
\hline \multicolumn{3}{|l|}{ E/e' alterado } \\
\hline$E / e^{\prime}<13(\%)$ & 93,94 & $p<0,01$ \\
\hline$E / e^{\prime}<8(\%)$ & 57,56 & $p<0,01$ \\
\hline VAI < 32ml/m2 (\%) & $71,1 \%$ & $p<0,01$ \\
\hline APS $\leq 35 \mathrm{mmHg}(\%)$ & $92 \%$ & $p<0,01$ \\
\hline \multicolumn{3}{|l|}{$E / e^{\prime}<13+$} \\
\hline \multicolumn{3}{|l|}{ VAl<32 ml/m2 + } \\
\hline PAPS $\leq 35 \mathrm{mmHg}(\%)$ & $64,8 \%$ & $p<0,01$ \\
\hline \multicolumn{3}{|c|}{ Sm: Velocidad de desplazamiento miocárdico en sístole } \\
\hline \multicolumn{3}{|c|}{ VAI: Volumen atrio izquierdo } \\
\hline \multicolumn{3}{|c|}{ PAPS: Presión sistólica de arteria pulmonar } \\
\hline
\end{tabular}

\section{Discusión}

No existió relación entre valores de Sm y FE en este estudio, lo que difiere con lo reportado en la literatura ${ }^{10}{ }^{12}$. Alam, et al. estudiaron a 78 pacientes post infarto agudo al miocardio y observaron una importante correlación entre los valores de $\mathrm{Sm}$ y la FE. Es más, valores de $\mathrm{Sm}>7,5$ $\mathrm{cm} / \mathrm{s}$ presentaron una sensibilidad de $79 \%$ y especificidad de $88 \%$ para predecir FE conservada ${ }^{12}$. Lo anterior se explica por el rol desempeñado por las fibras subendocárdicas, encargadas del acortamiento longitudinal durante la sístole. Una alteración a este nivel, tal como un IAM, podría incidir directamente en la $\mathrm{FE}^{13}$. Si se considera que la población incluida en nuestro estudio tiene FE preservada (promedio 66\%), es esperable que exista discrepancia con lo reportado en la literatura. $\mathrm{Al}$ no incluir pacientes con FE $<50 \%$ no es posible sacar conclusiones acerca de su asociación.

La asociación observada entre el IMV y los valores de Sm sustenta la hipótesis de que la alteración de la onda Sm podría ser un marcador precoz de HVI. Esto ha sido demostrado en pacientes con miocardiopatía hipertrófica14, sin embargo, hay poca evidencia acerca del valor para predecir cardiopatía hipertensiva ${ }^{7,15,16}$. Borges, et al. demostraron una significativa relación entre la disminución de los valores de Sm y aumento del IMV, y al mismo tiempo observaron valores de Sm disminuidos en pacientes hipertensos con mínima HVI, lo que podría predecir deterioro precoz de la función sistólica ${ }^{16}$.

La utilidad del DT en disfunción diastólica es ampliamente aceptada. No obstante, esto es válido para la velocidad de desplazamiento miocárdico en diástole (incluyendo la relación E/e'). Valores alterados de E/e' han mostrado ser el índice del DT con mayor impacto en la mortalidad de causa cardíaca ${ }^{17}$, por lo que es el parámetro recomendado para determinar la PFD en pacientes con FE conservada 9 . Menos estudiado es el valor de la onda Sm en disfunción diastólica. En nuestro trabajo se observa Sm alterada en el $71,2 \%$ de los pacientes con E/e' $\geq 13$ y se asocia a PFD aumentada. El 64,8\% de asociación entre valores de Sm normal y PFD conservada, podría convertir a la onda Sm en una herramienta complementaria para mejorar la estimación de la PFD en pacientes con E/e' entre 9-13, donde la asociación con E/e' no es tan clara.

De acuerdo a nuestros resultados y a lo publicado en la literatura, la medición de la onda Sm podría mejorar la valoración ecocardiográfica de pacientes hipertensos con FE conservada, ya que sería un sensible marcador de deterioro de la función sistólica inicial, especialmente en aquellos pacientes en que aparentemente estaría preservada, tales como disfunción diastólica. Conjuntamente, su asociación demostrada a HVI inicial y establecida, eventos cardiovasculares adversos y disfunción diastólica respalda aun 
más su utilidad en la práctica clínica.

\section{Conclusión}

La medición de la onda Sm aporta valiosa información a la evaluación del paciente hipertenso con FE conservada, pues se relaciona no solamente con HVI, sino también con parámetros de aumento de presión de fin de diástole.

Una limitación de este trabajo es que no disponemos del tiempo de evolución del diagnóstico de HTA.

Se requieren estudios prospectivos en nuestro medio para validar y confirmar los hallazgos y su uso en la práctica clínica.

\section{Referencias}

1. LAWES CM, VANDER HOORN S, RODGERS A. Internacional Society of Hypertension. Global burden of blood-pressurerelated disease, 2001. Lancet. 2008; 371:1513-8.

2. GOBIERNO DE CHILE. Ministerio de Salud. II Encuesta Nacional de Salud ENS 2009-2010, Chile.

3. WANG M, YIP G, WANG A, ZANG Y, HO P, TSE M, et al. Peak early diastolic mitral annulus velocity by tissue Doppler imaging adds independent and incremental prognostic value. $\mathrm{J}$ Am Coll Cardiol 2003; 41: 820-6.

4. SHIN HW, KIM H, SON J, YOON HJ, PARKS HS, CHO YK, et al. Tissue Doppler imaging as a prognostic marker for cardiovascular events in heart failure with preserved ejection fraction and atrial fibrillation. J Am Soc Echocardiogr 2010; 23: 755-61.

5. NIKITIN N, LOH P, DE SILVA R, GHOSH J, KHALEVA

$\mathrm{O}$, GOODE K, et al. Prognostic value of systolic mitral annular velocity measured with Doppler tissue imaging in patients with chronic heart failure caused by left ventricular systolic dysfunction. Heart 2006; 92: 775-9.

6. HAMEED A, GOSAL T, FANG T, AHMADIE R, LYTWYN M, BARAC I, et al. Clinical utility of tissue Doppler imaging in patients with acute myocardial infarction complicated by cardiogenic shock. J Cardiovasc Ultrasound 2008; 6:11.

7. WANG M, YIP G, WANG A, ZHANG Y, HO P, TSE M, et al. Tissue Doppler imaging provides incremental prognostic value in patients with systemic hypertension and left ventricular hypertrophy. J Hypertens 2005; 23: 183-91.

8. RIVAS-GOTZC,MANOLIOS M,THOHAN V,NAGUEH SF Impact of left ventricular ejection fraction on estimation of left ventricular filling pressures using tissue Doppler and flow propagation velocity. Am J Cardiol 2003; 91: 780-4.

9. NAGUEH S, APPLETON C, GILLEBERT T, MARINO P, OH J, SMISETH O, et al. Recommendations for the Evaluation of
Left Ventricular Diastolic Function by Echocardiography. Eur J Echocardiography 2009; 10: 165-193.

10. SANDERSON JE. HEART FAILURE WITH A NORMAL EJECTION FRACTION. Heart 2007; 93: 155-8.

11. PARK YS, PARK JH, AHN KT, JANG WI, PARK HS, KIM $\mathrm{JH}$, et al. Usefulness of Mitral Annular Systolic Velocity in the Detection of Left Ventricular Systolic Dysfunction: Comparison with Three Dimensional Echocardiographic Data. J Cardiovasc Ultrasound 2010; 18: 1-5.

12. ALAM M, WARDELL J, ANDERSSON E, SAMAD BA, NORDLANDER R. Effects of first myocardial infarction on left ventricular systolic and diastolic function with the use of mitral annular velocity determined by pulsed wave Doppler tissue imaging. J Am Soc Echocardiogr 2000; 13: 343-52.

13. HENEIN MY, GIBSON DG. Long axis function in disease. Heart 1999; 81: 229-31.

14. NAGUEH SF, BACHINSKI LL, MEYER D, HILL R, ZOGHBI W, TAM J, et al. Tissue Doppler imaging consistently detects myocardial abnormalities in patients with hypertrophic cardiomyopathy and provides a novel means for an early diagnosis before and independently of hypertrophy. Circulation 2001; 104: 128 -30 .

15. CUSPIDI C, NEGRI F, SALA C, VALERIO C, MANCIA G. Association of left atrial enlargement with left ventricular hypertrophy and diastolic dysfunction: A tissue Doppler study in echocardiographic practice. Blood Pressure 2012; 21: 24-30.

16. BORGES M, COLOMBO R, GONCALVES J, FERREIRA J, FRANCHINI K. Longitudinal Mitral Annulus Velocities Are Reduced in Hypertensive Subjects With or Without Left Ventricle Hypertrophy. Hypertension 2006; 47: 854-60.

17. WANG M, YIP G, YU CM, ZHANG Q, ZHANG Y, TSE D, et al. Independent and incremental prognostic value of early mitral annulus velocity in patients with impaired left ventricular systolic function. J Am Coll Cardiol 2005; 45: 272-7. 